\title{
COVID-19 Related Mortality Profile at a Tertiary Care Centre: a Descriptive Study
}

\author{
Sudhir Bhandari, ${ }^{1}$ Raman Sharma, ${ }^{1}$ Ajit Singh Shaktawat, ${ }^{1}$ Subrata Banerjee, ${ }^{1}$ \\ Bhoopendra Patel, ${ }^{2}$ Amit Tak, ${ }^{4}$ Deepa Meena, ${ }^{3}$ Abhishek Agarwal, ${ }^{1}$ Vishal Gupta, ${ }^{1}$ \\ Shrikant Sharma, ${ }^{1}$ Sunil Mahavar, ${ }^{1}$ Radhey Shyam Chejara, ${ }^{1}$ Govind Rankawat, ${ }^{1}$ Kapil \\ Gupta, ${ }^{4}$ Jitendra Gupta, ${ }^{4}$ Amitabh Dube, ${ }^{4}$ Shivankan Kakkar ${ }^{5}$
}

\section{Abstract}

Background/Aim: The recent pandemic of Severe Acute Respiratory Syndrome Corona Virus-2 (SARS-CoV-2) is yet another scourge from the coronaviridae family that causes illnesses ranging from common cold to more severe diseases such as Middle East respiratory syndrome (MERS-CoV) and severe acute respiratory syndrome (SARS-CoV). The numbers are still on the rise, despite a country wide lockdown and yet no definitive drugs and or/vaccines are available to manage the active COVID-19 cases.

Methods: The present research design was a hospital based observational descriptive study conducted at S. M. S. Medical College and Attached hospitals, Jaipur, that analysed data of all the patients with COVID-19 related mortality, admitted between 1 April to 4 May 2020. Patients included in this study were RT-PCR confirmed cases of SARS-CoV-2 using nasopharyngeal and oropharyngeal swab samples.

Results: The mean age of patients with COVID-19 related mortality was $53.41 \pm 18.42$ year with majority of patients belonging to age group of more than 60 years $(41.18 \%)$ followed closely by COVID-19 positive patients in age range of 45 to 60 years (33.33 $\%)$. The male to female ratio was 1.68: 1 . Mean time lag between hospitalization and death reported was 6.18 days. Majority of the patients admitted $(72.5 \%)$ succumbed within 3 days of hospitalization. Eleven patients $(21 \%)$ were brought dead to the hospital who were tested COVID-19 positive after death. Most common comorbidity reported in patients with COVID-19 related mortality was hypertension (30\%) followed by diabetes mellitus (27.5\%).

Conclusion: Hypertension and diabetes mellitus might be independent risk factors making an individual susceptible to COVID-19 related death. Elderly patients also have a greater risk of mortality. The non-availability of definitive management protocol and/or vaccine against COVID-19 makes public health preventive measures of social distancing, use of masks and frequent handwashing an important modality in the fight against COVID-19.

Key words: age, comorbidity, COVID-19, hypertension, mortality.
(1) Department of Medicine, S.M.S. Medi cal College \& Attached Hospitals, Jaipur (Rajasthan), India

(2) Department of Physiology, Government Medical College, Barmer (Rajasthan), India

(3) Department of Medical, Health and Family Welfare Services, Government of Rajasthan, India

(4) Department of Physiology, S.M.S. Medical College \& Attached Hospitals, Jaipur (Rajasthan), India

(5) Department of Pharmacology, S.M.S. Medical College \& Attached Hospitals, Jaipur (Rajasthan), India

Correspondence:

Bhoopendra Patel

E: bhupendra.kool9999@gmail.com

\section{ARTICLE INFO}

Received: 17 June 2020

Revision received: 27 June 2020 Accepted: 28 June 2020

\section{Introduction}

The recent pandemic of Severe Acute Respiratory Syndrome Corona Virus-2 (SARS-CoV-2) also known as Corona Virus Disease 2019 (COVID-19) is yet another scourge from the Coronaviridae family that causes illnesses ranging from common cold to more severe diseases such as Middle East respiratory syndrome (MERS-CoV) and severe acute respiratory syndrome (SARS-CoV). 
SARS-CoV-2 is a novel coronavirus that has not been previously identified in humans. The current pandemic of COVID-19 has affected 215 countries, areas, or territories worldwide as of 8 May 2020, and has infected 3,822,382 people worldwide, causing 263,658 confirmed deaths. ${ }^{1}$ The spread of COVID-19 began from Wuhan, a city of Hubei province of China and was declared a pandemic by World Health Organization (WHO) on 11 March $2020 .^{2}$

The clinical spectra of COVID - 19 is varied ranging from mild to moderate symptoms of cough, sore throat, headache, rhinorrhoea, vomiting and diarrhoea, fever and shortness of breath to signs and symptoms complex of severe pneumonia, acute respiratory distress syndrome, septic shock and/or multiple organ failure. ${ }^{3}$ The disease is highly infectious with a reproductive number (R0) ranging from 2.2-3.5, that explains its rapid spread like wild fire throughout the world. ${ }^{4}$

India has been struggling to contain the spread of virus and has managed to flatten the curve at 41,472 active cases and 2,109 deaths as of 10th May 2020, since the first reported SARS-CoV-2 case on 30 January $2020 .{ }^{5}$ The numbers are still on the rise, despite a country wide lockdown with yet no definitive management protocol inclusive of drugs and or/vaccines available to manage the active COVID-19 cases. State of Rajasthan is among the top five states of India with 3,708 confirmed cases of COVID-19 and 106 deaths reported till now. The present study was undertaken to appreciate and describe mortality profile of SARS-CoV-2 at one of premier Institute of Tertiary Care Medical College of Rajasthan and SouthEast Asia.

\section{Methods}

The present study, a hospital based observational descriptive study, was conducted at SMS Medical College and attached hospitals, Jaipur, sharing the highest load of patients in the Rajasthan that analysed and evaluated data of all COVID-19 related mortality of patients admitted between 1 April to 4 May 2020. A total of 51 mortalities were reported due to COVID-19 during this duration at this institute. All the patients were reverse - transcription polymerase chain reaction (RT - PCR) positive for SARS-CoV-2 using nasopharyngeal and oropharyngeal swab samples, tested at the
Laboratory of Microbiology of the Institute. The data where collected and analysed preserving the anonymity of patients. Patients were categorised into five different age groups to evaluate the relation between age and COVID-19 related mortality. Patients were also categorized in three groups based upon the number of days stayed in hospitals. The COVID-19 patients were also categorised based upon the underlying comorbidities to evaluate its relation with COVID-19 related mortalities. Data was presented and compared as mean and percentage of distribution among different groups.

\section{Results}

During a period of 34 days from 1st April to 4th May 2020 there were 51 deaths reported due to COVID-19. The mean age of patients with

Table 1: Characteristics of patients with COVID-19 related mortality

\begin{tabular}{|c|c|c|}
\hline & $\begin{array}{r}\text { Number of patients } \\
\text { with COVID-19 } \\
\text { related mortality }\end{array}$ & Percent \\
\hline \multicolumn{3}{|l|}{ Age Group (years, $n=51$ ) } \\
\hline $0-15$ & 1 & 1.96 \\
\hline $15-30$ & 8 & 15.67 \\
\hline $30-45$ & 4 & 7.84 \\
\hline $45-60$ & 17 & 33.33 \\
\hline$>60$ & 21 & 41.18 \\
\hline \multicolumn{3}{|l|}{ Gender $(n=51)$} \\
\hline Female & 19 & 37.26 \\
\hline Male & 32 & 62.74 \\
\hline \multicolumn{3}{|c|}{ Hospital stay group (days, $n=40$ ) } \\
\hline $1-3$ & 29 & 72.5 \\
\hline $4-6$ & 6 & 15 \\
\hline$>6$ & 5 & 12.5 \\
\hline \multicolumn{3}{|l|}{ Comorbidity $(n=40)$} \\
\hline No comorbidity & 4 & 10 \\
\hline Hypertension & 12 & 30 \\
\hline Diabetes Mellitus & 11 & 27.5 \\
\hline Chronic Kidney Disease & 2 & 5 \\
\hline Cardiovascular Disease & 6 & 15 \\
\hline Respiratory Disease & 6 & 15 \\
\hline Gastrointestinal Disease & 4 & 10 \\
\hline Sepsis & 6 & 15 \\
\hline MODS & 3 & 7.5 \\
\hline \multicolumn{3}{|l|}{ Brought in status $(n=51)$} \\
\hline Brought Dead & 11 & 21.57 \\
\hline Brought alive & 40 & 78.43 \\
\hline
\end{tabular}

MODS = Multiple organ dysfunction syndrome 
COVID-19 related mortality was $53.41 \pm 18.42$. The highest mortalities ( $\mathrm{n}=21,41.18 \%$ ) were reported in the group above 60 years of age, closely followed by $45-60$ years age group $(n=17,33.33$ $\%)$. The lowest mortality was observed in paediatric age group below 15 years with overall one death $(1.96 \%)$. Males $(\mathrm{n}=32,62.74 \%)$ were affected more than the females $(n=19,37.26 \%)$ with a male to female ratio of $1.68: 1$. One death was reported in pregnant female positive for COVID-19. (Table 1).

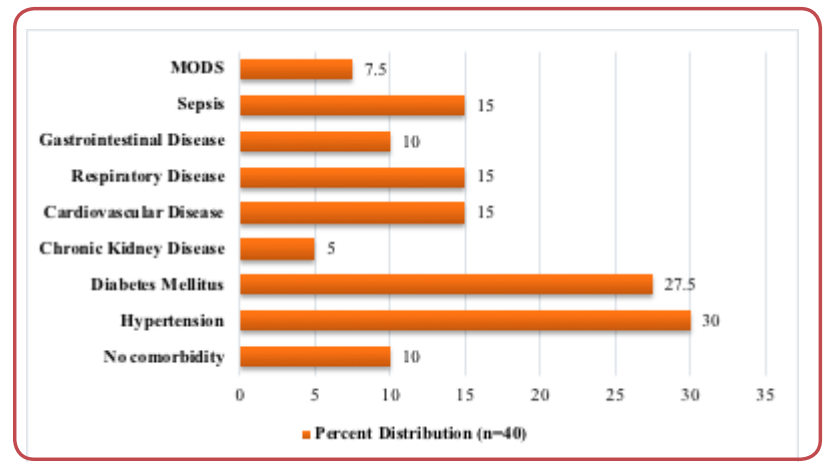

Figure 1: Comorbidities in patients with COVID-19 related mortality

MODS = Multiple organ dysfunction syndrome

Majority of patients ( $\mathrm{n}=29,72.5 \%$ ) succumbed within a duration of 1-3 days during hospital stay. Six patients (15\%) survived for a duration of 4-6 days and only five patients (12.5\%) could survive for more than six days. The Mean time lag between hospitalization and death reported was 6.18 days. The comorbidity most prevalent in patients with COVID-19 related mortality was hypertension ( $\mathrm{n}=12,30 \%)$, closely followed by diabetes mellitus ( $\mathrm{n}=11,27.5 \%$ ). Other comorbidities such as cardiovascular diseases $(\mathrm{n}=6,15$ $\%)$, respiratory disease ( $\mathrm{n}=6,15 \%)$, sepsis $(\mathrm{n}=$ 6, $15 \%)$, gastrointestinal disease $(n=4,10 \%)$, multiple organ dysfunction syndrome $(\mathrm{n}=3,7.5$ $\%)$ and chronic kidney disease ( $\mathrm{n}=2,5 \%)$. Four patients (10\%) with COVID-19 related mortality did not have any associated comorbidities. There were 11 patients $(21.57 \%)$ who were brought dead and were reported positive for SARS-CoV-2 post mortem (Table 1 and Figure 1).

\section{Discussion}

The disease spectrum of COVID-19 may very from a mild illness to fatal complications like pneumonia, acute respiratory distress syndrome (ARDS), multi-organ failure, septic shock, disseminated intravascular coagulation and ultimately leading to death. ${ }^{6,7}$ Outcome of COVID-19 may be poorer in case of underlying comorbidities. ${ }^{8}$ Therefore, identification of such underlying factors is of paramount importance in COVID-19 management. In the present study the mean age of patients with COVID-19 related mortality was $53.41 \pm 18.42$, suggesting a higher mortality among elderly individuals. Chen et $\mathrm{al}^{9}$ also observed a similar average age profile of COVID-19 positive patients in the median age of 55.5.

Majority of patients afflicted with COVID-19 belonged to the age group of more than 60 . Several studies have observed this association, indicating elderly age contributing to a severe outcome..$^{10-13}$ An elderly individual might be susceptible to COVID-19 due to age-related changes in pulmonary functions with obvious responsiveness and tolerance, subsequently leading to poor outcome in this age group. ${ }^{14}$ COVID-19 exhibited preponderance for male individuals with a male to female ratio of $1.68: 1$, a finding that has been reported in various studies worldwide. ${ }^{3,15}$ Jin et $\mathrm{al}^{16}$ also observed that the number of men with COVID-19 related mortality was 2.4 times higher than that of women.

In the present study hypertension was the most prevalent comorbidity in COVID-19 related mortality, closely followed by diabetes mellitus. This indicates a higher mortality risk in COVID-19 patients presenting with hypertension, followed by diabetes mellitus, a finding that supplants the observations of Guan et al from China ${ }^{15}$ and Itelman et al from Israel, ${ }_{1}^{17}$ supporting the premise that chances of morbidity and mortality in COVID-19 positive patients increase with associated comorbid conditions. Hypertension has been suggested as a risk factor for poor outcome in COVID-19 patients in several studies. ${ }^{12,13,18}$ A similar finding in hypertensives was also observed in the present study. The exact mechanism underlying this observation is still unclear. However, the use of angiotensin-converting enzyme (ACE) inhibitors and angiotensin receptor blockers used for treatment of hypertension has been linked to severe outcome in such patients. These drugs have been found to augment ACE2 mRNA expression. ${ }^{19}$ SARS-CoV-2 has been found to utilis e this ACE2 receptor for invading the human cells. ${ }^{20} \mathrm{~A}$ higher expression of these receptors facilitates entry of this virus, increasing the susceptibility of individuals to this infection and subsequently a 
severe disease. This receptor not only acts as the entry receptor of SARS-CoV-2, but also protects the lung from injury due to its anti-inflammatory effects. ${ }^{21}$

ACE2 exhibits anti-inflammatory and protective role that has been found downregulated in diabetics. ${ }^{22}$ The downregulated anti-inflammatory response could be responsible for exaggerated immune response to SARS-CoV-2 virus in diabetics, with a severe and uncontrolled damage to the lungs and other tissues. This might explain the high mortality in diabetics observed in the present study. Respiratory and other cardiovascular diseases were also observed in patients with COVID-19 related mortality in this study. Acute respiratory distress syndrome with extensive inflammation, cell death, alveolar damage and oedema occurs in severe COVID-19 case leading to a hypoxic state due to reduced gaseous exchange. . $3,24^{24}$ This could explain the possible severe outcome in patients with pre-existing respiratory diseases, as has been observed in this study.

Mortality in COVID-19 patients with cardiovascular diseases observed in this study could be attributable to a wide expression of ACE2 in cardiovascular system that is more pronounced in cardiovascular disease. ${ }^{25,}{ }^{26}$ Several researchers have pointed out the myocardial damage and related mortality in patients of SARS-CoV-2 infection. ${ }^{3,27,28}$ In the present study around 3/4 of the patients succumbed within 3 days of hospitalization and surprisingly $1 / 5$ of the patients was brought dead, who were tested positive post mortem. This finding indicates a rapid progression of COVID-19 in these patients.

The disease profile of COVID-19, inclusive of its behaviour, progress and severity scale, is crucial to determine appropriateness and adequacy of mitigation strategies and to enable planning and designing health-care needs and policies. Moreover, factors that might contribute to rapid progression of COVID-19, such as virulence, drug resistance, host factors or structural reformation of the virus, needs to be explored and should form the epicentre of focus especially in people without any risk factors. Country wide lockdown and social distancing has helped in containing the spread of virus to some extent, but the stumbling economy due to lock down poses a great challenge worldwide.

\section{Conclusion}

Hypertension and diabetes mellitus might be independent risk factors making an individual susceptible to COVID-19 related death. Elderly patients also have a greater risk of mortality. The non-availability of definitive management protocol and/or vaccine against COVID-19 makes public health preventive measures of social distancing, use of masks and frequent handwashing an important modality in the fight against COVID-19.

\section{Acknowledgements}

The contributors of the article acknowledge invaluable inputs of Mr. Rohit Kumar Singh (Additional Chief Secretary), Mr. Vaibhav Galriya (Secretary, Medical Education), Mr. Ravi Sharma (Additional Director, Department of Medical \& Health) from Government of Rajasthan, Departments of Medicine, Community Medicine and Microbiology of S.M.S. Medical College and Attached Hospitals, Jaipur and Government of Rajasthan, for their ongoing support against the menace of global pandemic of COVID - 19.

\section{Conflict of interest}

None.

\section{References}

1. World Health Organization. Coronavirus disease (COVID-19) pandemic

Available from: https://www.who.int/emergencies/ diseases/novel-coronavirus-2019. Accessed on 8 May 2020.

2. World Health Organization. Statement on the second meeting of the International Health Regulations (2005) Emergency Committee regarding the outbreak of novel coronavirus (2019-nCoV). Availabe from: https:// www.who.int/news-room/detail/30-01-2020-statement-on-the-second-meeting-of-theinternational-health-regulations-(2005)-emergency-committee-regarding-the-outbreak-ofnovel-coronavirus-(2019-ncov). Accessed on 8 May 2020.

3. Huang C, Wang Y, Li X, Ren L, Zhao J, Hu Y,et al. Clinical features of patients infected with 2019 novel coronavirus in Wuhan, China. Lancet 2020;395: 497 - 506.

4. Zhao S, Lin Q, Ran J, Musa SS, Yang G, Wang W, et al. Preliminary estimation of basic reproduction number of 
novel coronavirus (20192-nCov) in China, from 2019 to 2020: a data-driven analysis in the early phase of the outbreak. Int I Infect Dis 2020; 92:214-7.

5. COVID-19 India. Available from: https://www.mohfw. gov.in/. Accessed on 8 May 2020.

6. Hui DS, Azhar E, Madani TA, Ntoumi F, Kock R, Dar O, et al. The continuing 2019-nCoV epidemic threat of novel coronaviruses to global health - The latest 2019 novel coronavirus outbreak in Wuhan, China. Int J Infect Dis 2019;91:264-6.

7. Murthy S, Gomersall CD, Fowler RA. Care for critically ill patients with COVID-19. JAMA 2020;323(15):1499-500.

8. Wu Z, McGoogan JM. Characteristics of and important lessons from the coronavirus disease 2019 (COVID-19) outbreak in China: summary of a report of 72,314 cases from the Chinese Center for Disease Control and Prevention. JAMA 2020;323(13):1239-42.

9. Chen N, Zhou M, Dong X, Qu J, Gong F, Han Y, et al. Epidemiological and clinical characteristics of 99 cases of 2019 novel coronavirus pneumonia in Wuhan, China: a descriptive study. Lancet 2020;395(10223):507-13.

10. Liu Y, Mao B, Liang S, Yang JW, Lu HW, Chai YH, et al. Association between age and clinical characteristics and outcomes of COVID-19. Eur Respir J 2020 May 27;55(5):2001112. doi: 10.1183/13993003.011122020.

11. Yang X, Yu Y, Xu J, Shu H, Xia J, Liu H, et al. Clinical course and outcomes of critically ill patients with SARS-CoV-2 pneumonia in Wuhan, China: a single-centered, retrospective, observational study. Lancet Respir Med 2020;8(5):475-81.

12. Zhou F, Yu T, Du R, Fan G, Ying L, Liu Z, et al. Clinical course and risk factors for mortality of adult inpatients with COVID-19 in Wuhan, China: a retrospective cohort study. Lancet 2020;395(10229):1054-62.

13. Wu C, Chen X, Cai Y, Xia J, Zhou X, Xu Sha, et al. Risk factors associated with acute respiratory distress syndrome and death in patients with coronavirus disease 2019 pneumonia in Wuhan, China. JAMA Intern Med 2020 Mar 13;e200994. doi: 10.1001/jamainternmed.2020.0994.

14. Miller EJ, Linge HM. Age-related changes in immunological and physiological responses following pulmonary challenge. Int J Mol Sci 2017 Jun; 18(6):1294. doi:10.3390/ijms18061294.

15. Guan $W$-jie, Liang $W$-hua, Zhao $Y$, Liang $H$, Chen $Z$, $\mathrm{Li}$ Y, et al. Comorbidity and its impact on 1590 patients with Covid-19 in China: a nationwide analysis. Eur Respir J 2020 May 14;55(5):2000547. doi: 10.1183/13993003.00547-2020.

16. Jin J-M, Bai P, He W, Wu F, Liu X-F, Han D-M, et al. Gender differences in patients with COVID-19: focus on severity and mortality. Front Public Health 2020 Apr 29;8:152. doi: 10.3389 /fpubh.2020.00152.

17. Itelman E, Wasserstrum Y, Segev A, Avaky C, Negru L, Cohen $\mathrm{D}$, et al. Clinical characterization of 162 patients in Israel: preliminary report from a large tertiary center. Isr Med Assoc J 2020;22:271-4.
18. Chen T, Wu D, Chen H, Yan W, Yang D, Chen G, et al. Clinical characteristics of 113 deceased patients with coronavirus disease 2019: retrospective study. BMJ 2020 Mar 26;368:m1091. doi: 10.1136/bmj.m1091.

19. Ferrario CM, VonCannon, J, Jiao Y, Ahmad S, Bader M, Dell'Italia LJ, et al. Cardiac angiotensin-(1-12) expression and systemic hypertension in rats expressing the human angiotensinogen gene. Am J Physiol Heart Circ Physiol 2016 Apr 15;310(8):H995-1002.

20. Hoffmann M, Kleine-Weber H, Schroeder S, Krüger N, Herrler T, Erichsen S, et al. SARS-CoV-2 cell entry depends on ACE2 and TMPRSS2 and is blocked by a clinically proven protease inhibitor. Cell 2020 Apr 16;181(2):271-80.

21. Zhang HA, Penninger JM, Li Y, Zhong N, Slutsky AS. Angiotensin-converting enzyme 2 (ACE2) as a SARS-CoV-2 receptor: molecular mechanisms and potential therapeutic target. Intensive Care Med 2020 Apr;46(4):586-90.

22. Tikellis $C$, Thomas MC. Angiotensin-converting enzyme 2 (ACE2) is a key modulator of the renin angiotensin system in health and disease. Int J Pept 2012;2012:256294. doi: $10.1155 / 2012 / 256294$.

23. Zangrillo A, Beretta L, Scandroglio AM, Monti G, Fominskiy E, Colombo S, et al. Characteristics, treatment, outcomes and cause of death of invasively ventilated patients with COVID-19 ARDS in Milan, Italy. Crit Care Resusc 2020 Apr 23. Online ahead of [published online ahead of print]. Avaliable from: https://pubmed.ncbi. nlm.nih.gov/32353223/. Accessed on 27 June 2020.

24. Diamond M, Peniston Feliciano HL, Sanghavi D, Mahapatra S, et al. Acute Respiratory Distress Syndrome (ARDS) [Updated 2020 Jan 5]. In: StatPearls [Internet]. Treasure Island (FL): StatPearls Publishing; 2020 Jan. Available from: https://www.ncbi.nlm.nih.gov/books/ NBK436002/. Accessed on: 20 June 2020.

25. Chen L, Li X, Chen M, Feng Yi, Xiong C. The ACE2 expression in human heart indicates new potential mechanism of heart injury among patients infected with SARS-CoV-2. Cardiovasc Res 2020 May 1;116(6):1097-100.

26. Guo J, Huang Z, Lin L, Lv J. Coronavirus Disease 2019 (COVID-19) and cardiovascular disease: a viewpoint on the potential influence of angiotensin-converting enzyme inhibitors/angiotensin receptor blockers on onset and severity of severe acute respiratory syndrome coronavirus 2 infection. J Am Heart Assoc 2020 Apr 7;9(7):e016219. doi: 10.1161/JAHA.120.016219.

27. Wang D, Hu B, Hu C, Zhu F, Li X, Zhang J, et al. Clinical characteristics of 138 hospitalized patients with 2019 novel coronavirus-infected pneumonia in Wuhan, China. JAMA 2020;323(11):1061-9.

28. Ruan, Q Yang, K, Wang, W, Jiang L, Song J. Clinical predictors of mortality due to COVID-19 based on an analysis of data of 150 patients from Wuhan, China. Intensive Care Med 2020 May;46(5):846-8. 